\title{
Mathematical modeling for resting state functional connectivity of cortical and sub-cortical networks
}

\author{
Mohit H Adhikari ${ }^{1 *}$, Alessandra Griffa ${ }^{2,3}$, Patrick Hagmannn ${ }^{2,3}$, Maurizio Corbetta ${ }^{4}$, Gustavo Deco ${ }^{1}$ \\ From Twenty Second Annual Computational Neuroscience Meeting: CNS*2013 \\ Paris, France. 13-18 July 2013
}

Mathematical modeling studies for brain activity at rest have mainly focused on networks of cortical areas. However, cortical areas are connected to sub-cortical areas, such as the thalamus and the hippocampus, whose contribution to the resting state activity cannot be ignored. Further, modeling for diseases such as stroke, in which the focus is usually in a sub-cortical region, requires taking sub-cortical networks into consideration.

Here, we use the structural connectivity (SC) matrix of 83 areas (68 cortical, 15 sub-cortical) from 10 subjects, obtained using diffusion spectrum imaging, to model the activity of these regions. Each node of this network is modeled using the dynamical mean field model of Wong and Wang [1,2] involving a single stochastic differential equation for the population firing rate. In the absence of coupling, each area is in the spontaneous state of low firing rate. Next, we simulate deterministic dynamical equations for the first and second order statistical moments of this stochastic system and obtain simulated functional connectivity (sFC). We then vary three coupling parameters - wc, wsc and wcsc - multiplying the SC strengths between (a) cortical areas, (b) sub-cortical areas and (c) cortical and sub-cortical areas respectively and compare the $\mathrm{sFC}$ with the empirical functional connectivity (FC) during resting state. We also calculate the bifurcation diagram of the network as a function of these three parameters.

In this three-dimensional parameter space we obtain the maximum correlation between $\mathrm{FC}$ and $\mathrm{sFC}$ values for both cortical and sub-cortical areas near the bifurcation surface where the spontaneous state loses stability with higher values of $\mathrm{w}_{\mathrm{c}}$ and $\mathrm{w}_{\mathrm{csc}}$ compared to $\mathrm{w}_{\mathrm{sc}}$. Interestingly, in this region, while the sFC for cortical areas correlates well with the corresponding $\mathrm{SC}$ values, it does not do so for sub-cortical areas. This suggests that the functional connectivity between sub-cortical areas during the resting state is influenced more by their anatomical connections with the cortical areas than between themselves. It also validates our choice of using three coupling parameters instead of just one global coupling parameter. We aim to use this study to model the resting state functional connectivity in patients of stroke.

\section{Author details}

Theoretical and Computational Neuroscience, Center for Brain and Cognition, University of Pompeu Fabra, Barcelona, 08018, Spain. ${ }^{2}$ Signal Processing Laboratory, Ecole Polytechnique Fédérale de Lausanne (EPFL), Lausanne, Switzerland. ' ${ }^{3}$ Department of Radiology, Lausanne University Hospital (CHUV) and University of Lausanne (UNIL), Lausanne, Switzerland. ${ }^{4}$ Washington University in St. Louis, School of Medicine, Department of Neurology, Radiology, Anatomy and Neurobiology, St. Louis, USA.

Published: 8 July 2013

\section{References}

1. Wong $K-F$, Wang $X-J$ : A recurrent network mechanism of time integration in perceptual decisions. J Neurosci 2006, 26(4):1314-1328.

2. Deco G, Ponce-Alvarez A, Mantini D, Romani GL, Hagmann P, Corbetta M: Resting-state functional connectivity emerges from structurally and dynamically shaped slow linear fluctuations., submitted for publication.

doi:10.1186/1471-2202-14-S1-P101

Cite this article as: Adhikari et al:: Mathematical modeling for resting state functional connectivity of cortical and sub-cortical networks. BMC Neuroscience 2013 14(Suppl 1):P101.

\footnotetext{
*Correspondence: mohit.adhikari@upf.edu

'Theoretical and Computational Neuroscience, Center for Brain and

Cognition, University of Pompeu Fabra, Barcelona, 08018, Spain

Full list of author information is available at the end of the article
}

(c) 2013 Adhikari et al; licensee BioMed Central Ltd. This is an Open Access article distributed under the terms of the Creative Commons 